\title{
Simulation of Gender Artificial Society: Multi-agent Models of Subject-Object Interactions
}

\author{
Julia Frolova ${ }^{1}$ and Victor Korobitsin ${ }^{1}$ \\ Omsk State University, Mathematical Modelling Chair, \\ 55A, Mira pr., 644077 Omsk, Russia \\ \{frolova, korobits\}@univer.omsk.su \\ http://www.univer.omsk.su/socsys
}

\begin{abstract}
In the paper is presented an approach to simulation of gender interaction in artificial society. We are studying the process of the male's and female's interaction based on subject-object relations. On the one hand, the females radiate the charm pulses. Responding to these pulses, the males choose a female. On the other hand, the males accumulate a resource and females choose a male, having the maximum resource wealth. The computer simulation were realized on $\mathcal{S W} \mathcal{A R \mathcal { M }}$. We reveal three types of agent interaction. These types is characterized by a subject-object interaction coefficient, defining the stability of agent interaction.
\end{abstract}

\section{Introduction}

The aim of research is a studying the behaviour of males and females and their differences, we research not only a man but also a society as a whole.

We consider a gender system in society. It differs from population by inclusion of gender structure. The existence of two types of agent (male and female) is needed under construction of artificial society model. We intend to add the gender structure in artificial society model for its adequacy to real society. Gender System is a collection of social relations between males and females that are defined their interpersonal interactions. The system of individual's connection has the informational structure, presented as a communication model of society.

Computer Simulation has the huge possibilities for understanding the society: the research of individuals, individual interactions, family, social group and organization, ethnoses, and social systems [2]. The computer models of gender systems help to develop the sociological approaches, reproducing and playing in real relations. Replacing the real gender relations by the computer model we come from the notion about gender as a special type of intrigue [3]. Both in intrigue and in discourse the source structure has a form of collection of simple sentences, connected between itself by various logical relations. The elements of discourse and intrigue are the events, participants and "non-events". The non-events include: the circumstances accompanying the events, the background 
explaining the events, the estimation of event participants, the information correlating discourse (or intrigue) with events.

Considering the gender system we are studying the process of the male's and female's interaction based on subject-object relations [4]. Each individual can be presented and as subject and as object of interaction. Defining the individual state, we describe the respective act of subject and the change of object state in artificial society.

Defining the gender as an intrigue allows easy to go to constructing the model of gender interaction in artificial society. This is because, model is the playing simulation, reproducing the parameters a close to intrigue (see Table 1).

Table 1. Gender as Intrigue vs Computer Model

\begin{tabular}{ll}
\hline \multicolumn{1}{c}{ Gender as Intrigue } & Computer Model \\
\hline - participants & $\bullet$ agents \\
- circumstances accompanying the events & • rules \\
- events & • agent interactions \\
- background explaining the events & $\bullet$ environment \\
- estimation of event participants & - interacting the agents \\
& with environment \\
- information correlating the intrigue & - change into environment \\
$\quad$ with events & \\
\hline
\end{tabular}

\section{Description of interaction process between males and females}

The communication process between males and females has a collection of main elements of interaction. The problem of the computer model is an attempt to select they.

Under gender contact, the people are interacting on relations of confidence, understanding, supports, respects, and imitations. But they are in condition of the fights with the individuals of the same sex. The interaction between the different sexes is built on delight and pulses for males and on material and emotional support for females. Assigning the even chance for agents of artificial society and limiting the environment for they, we create the competition for agents of same sex. For satisfying its need, the agent must overtake an adversary. Then he will has got the necessary interaction.

The males and females are the equivalent individuals but they have the various gender particularities.

On the one hand, the females have an unique device for influence on the male behaviour. Herewith the males have own system of perception. For example, the influence device is a beauty, glamour and etc. In this instance the female is 
defined as a subject of action and the male is an object of influence in gender relations. This definition is a foundation of the first presented model.

On the other hand, the males have the resource and females are consuming it. In this instance the female is defined as an object and the male is a subject of action in gender relations. This definition is a foundation of the second presented model.

The result of interaction process is characterized by a Gender Interaction Coefficient, given by

$$
\mathcal{G}=\frac{C_{f}}{F}: \frac{C_{m}}{M}
$$

where $F, M$ are numbers of females and males, respectively. $C_{f}, C_{m}$ are numbers of interacting females and males, respectively. This numbers are shown how much agents participate in the interaction process. Thus the Gender Interaction Coefficient is given by the relation of the interaction proportions of females and males. If $\mathcal{G}$ equals 1.0 then we can observe the interaction process with gender equality in artificial society. When $\mathcal{G}$ less than 1.0 then the interaction majority is formed by the males. If $\mathcal{G}$ more than 1.0 then the males participate in the interaction with lesser activity.

These models were realized on multi-agent modelling system $\mathcal{S W} \mathcal{A R \mathcal { M }}$ [1]. This system allow to program the artificial society, defining the respective parameters, rules of behaviour and interaction for agents and environment. Designed object-oriented $\mathcal{S} \mathcal{W} \mathcal{A} \mathcal{R} \mathcal{M}$ libraries allows to demonstrate the agents' behaviour on computer display in real time and to get necessary characteristics by graphs and diagrams.

\section{The first model}

The pulses of female charm are the power of action for attraction between males and females. The attraction is an important component of interpersonal attractiveness. It is very involved with a need of interaction one with other. The feminine influence possesses the different degree of intensity, defining power of influence. The male is presented in models as a creature, encircled with all sides by radiated pulses. But he can make choice, realizing the possibility of the individual liberty of movement. The field of gender interactions is formed by means of made choice. It is defined as a superposition of two personal spaces.

The personal space of any individual is characterized by the communication potential, reflecting the condition of the contact process. The communication potential is feature of the personal possibilities, defining the quality of the individual contact. Its level depends on a sociability, nature and toughness of contacts, installed with other people. We may consider the interpersonal attraction as a condition and result of compatibility of two persons under interaction. 


\subsection{Constructing model of pulse influence}

We apply the multi-agent modelling method for constructing model of pulse influence. Hereinafter we will describe the environment, agents, rule of movement and interaction for agents.

The Environment. The model describes the interaction of males and females inside the limited space (environment). We define the environment as an interaction field. Let the field be a square-wave area on plane. The interaction field wraps around from right to left (that is, were you to walk off the screen to the right, you would reappear at the left) and from top to bottom, forming a doughnut - technically, torus. We denote the model attributes:

$T$ is the maximum simulated time for the model;

$X, Y$ are the horizontal and vertical field dimensions, respectively.

Also, we denote the model variable:

$t$ is a time $(0 \leq t \leq T)$.

The Agents. We define the male as a $m$-agent, the female as a $f$-agent. At every time each agent has a position given by an ordered pair $(x, y)$ of horizontal and vertical lattice coordinates, respectively $(x \in[0, X], y \in[0, Y])$. Two agents are not allowed to occupy the same position. We shall investigate a random distribution of $M m$-agents and $F f$-agents in environment.

All agents have the own personal space and communication potential. The communication potential value is a variable state. Define it as $H_{f}(t)$ and $H_{m}(t)$ for $f$-agent and $m$-agent, respectively.

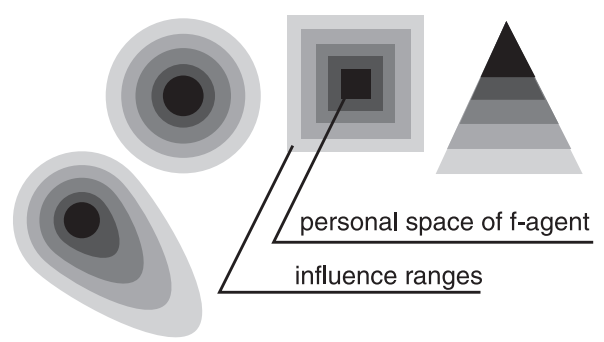

Fig. 1. An influence ranges

Each $f$-agent is source of pulse radiation and one has the influence range on $m$-agent. This area forms by radiation of charm pulses with various intensity level $I_{f}$. They can be the some forms included the personal space of $f$-agent. The maximum value of intensity achieves in this space and reduces with distribution on field of interaction. The intensity value is a variable state depended on communication potential level. The influence of $f$-agent on $m$-agent is reduced 
when this $m$-agent come away from source of pulse radiation. The interaction field is created through superposition of influence ranges of several $f$-agents. The example of areas is shown in figure 1.

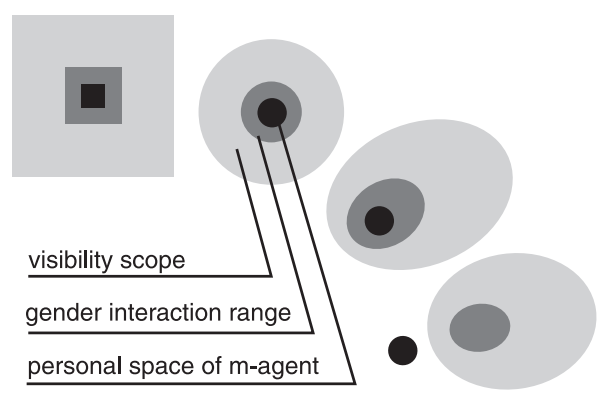

Fig. 2. An interaction ranges

Each $m$-agent has the individual system of perception. He look over the field of possible interaction within a some area defined as a visibility scope. This area can be the some form and not include the personal space of $m$-agent. Also, the gender interaction range is defined inside the visibility scope. The process of interaction between $m$-agent and $f$-agent can only occur $f$-agent within gender interaction range of $m$-agent. The example of ranges is shown in figure 2 .

The movement rule. The $m$-agents are also given a movement rule. From interaction field within the visibility scope of $m$-agent, find the nearest unoccupied position of maximum intensity of influence, go there.

The contact rule. The interaction process of agents occurs according to contact rule. If $f$-agent fall into the gender interaction range of $m$-agent then the interaction process is realizing. Under interaction process, the communication potential is being increased and the influence intensity is being decreased. In the absence of interaction process, the influence intensity is being increased and the communication potential is being decreased. For each $m$-agent

$$
H_{m}(t+1)=H_{m}(t)+\alpha N_{m}-\beta,
$$

where $\alpha$ is an increase rate of communication potential; $\beta$ is a decrease rate of one under interaction absence; $N_{m}$ is a number of $f$-agents interacting with this $m$-agent. The value $N_{m}$ is defined by the number of $f$-agents inhered in the interaction range of this $m$-agent. For each $f$-agent

$$
H_{f}(t+1)=H_{f}(t)+\alpha N_{f}-\beta,
$$

where $N_{f}$ is a number of $m$-agents interacting with this $f$-agent. 
The influence intensity is changed by the following rule

$$
I_{f}=\frac{I_{0}}{H_{f}+1},
$$

where $I_{0}$ is a maximum value of intensity.

\subsection{Computer simulation of interaction process based on the pulse influence}

We shall investigate a random distribution of $53 \mathrm{f}$-agents - the color is black and $47 \mathrm{~m}$-agents - the color is white, as shown in figure 3 , on the left.
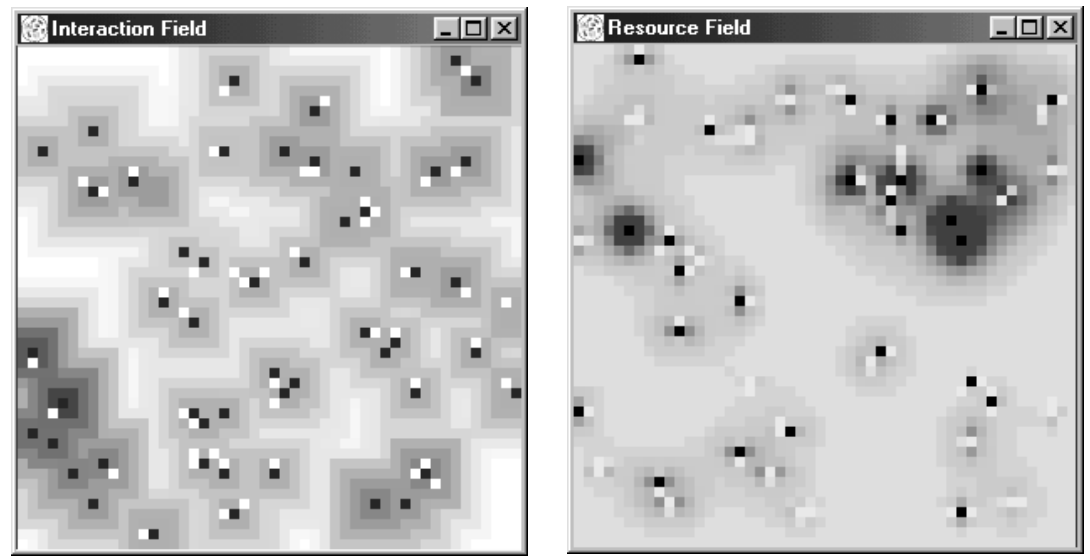

Fig. 3. Interaction field and Resource field

Environment consists of 2500 locations arranged on a $50 \times 50$ lattice with the influence intensity level at every site initially. The intensity score is highest at the peaks in the field around the cell occupied by $f$-agent - where the color is most black - and falls off in a series of terraces. The intensity scores range from some maximum - here $I_{0}=100$ - at the peaks to zero.

The value $\alpha$ (increase rate of communication potential) is set 0.015 and the value $\beta$ (decrease rate of communication potential) is set 0.01 . The visibility scope of $m$-agent, influence range of $f$-agent, and gender interaction range of $m$-agent have the square forms with linear sizes 11,17 , and 3 , respectively.

Describe the simulation process in dynamics. The $m$-agent is moving on the field according to movement rule, and fall into the influence range of some $f$ agent. The $m$-agent go to the cell with maximum value of influence intensity. When the $f$-agent fall into the gender interaction range of $m$-agent then the interaction process is realized. Under interaction process the agent characteristics are changed according to the contact rule. The result of interaction series is 
a decrease of influence level of $f$-agent. In consequence of which the $m$-agent continues the search of $f$-agent with high influence level. And the process of interactions is being replayed.

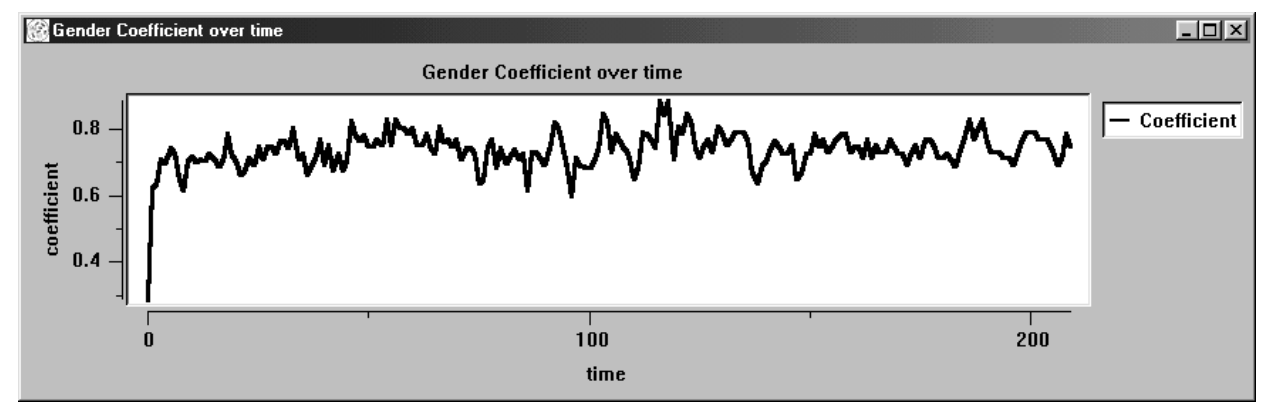

Fig. 4. Gender Interaction Coefficient value dynamics

On the figure 4 is presented the graph of Gender Interaction Coefficient value dynamics. $\mathcal{G}$ is fluctuating in interval $[0.6,0.9]$.

\section{The second model}

Similarly the first model, we use the multi-agent modelling method for constructing the model of resource consumption. We also denote the model attributes and variables, and lattice. The interaction of $m$-agent and $f$-agent is realized within the resource field. The field also has the form of torus. The $m$-agent accumulate the resource. The resource field is formed according to resource wealth of $m$-agent. The $f$-agent has a need in consumption of resource. Therefore he is moving for searching the $m$-agent, having maximum resource wealth.

\subsection{Constructing model of resource consumption}

The Environment. In this model we define the environment as a resource

field. Each $(x, y)$ cell is characterized by the attributes:

$\varepsilon$ is a rate of resource distribution;

$\beta_{0}(x, y)$ is a rate of resource depletion at cell $(x, y)$;

$\gamma_{0}(x, y)$ is a resource loss at cell $(x, y)$

and the state variables:

$u(x, y, t)$ is a level of resource at cell $(x, y)$ at time $t$;

$p_{m}(x, y, t)$ is a occupancy status of cell $(x, y)$ by $m$-agent at time $t$. This function has a value equaled 1.0 in case the cell is occupied by $m$-agent and 0.0 in other case;

$p_{f}(x, y, t)$ is a occupancy status of cell $(x, y)$ by $f$-agent at time $t$. This function has a value equaled 1.0 in case the cell is occupied by $f$-agent and 0.0 in other case. 
The Agents. Each $m$-agent is a source of resource, which change with time. He has the initial resource capacity. The initial resource forms the resource field at first time. The $m$-agent is characterized by the attributes:

$\beta_{m}$ is a rate of the resource accumulation;

$\gamma_{m}$ is a level of resource loss. It is set from interval $\left[\gamma_{\min }, \gamma_{\max }\right]$;

$\zeta_{m}$ is an additional resource bonus;

$s_{m}$ is an initial level of resource. It is set from interval $\left[s_{\min }, s_{\max }\right]$;

$\left(x_{m}, y_{m}\right)$ is a field cell occupied by the $m$-agent

and the state variable:

$w(t)$ is a amount of resource wealth at time $t$. Its value equals $\left.u\left(x_{m}, y_{m}, t\right)\right)$.

Each $f$-agent is characterized by the attributes:

$v$ is a visibility scope;

$\beta_{f}$ is a rate of the resource consumption

and the state variable:

$\left(x_{f}, y_{f}\right)$ is a field cell occupied by the $f$-agent.

The movement rule. The $f$-agents are given a movement rule, which is a kind of gradient search algorithm. From resource field within the visibility scope of $f$-agent, find the nearest unoccupied position of maximum level of resource, go there and consume the resource.

The rule of resource change on field. Under influence of agent's behaviour, the resource level in cell $(x, y)$ is being changed at time. The resource level is changed in the following events:

- if this cell occupied by $m$-agent then resource level is being increased at a rate $\beta_{m}$;

- if this cell occupied by $f$-agent then resource level is being decreased at a rate $\beta_{f}$;

- if this cell unoccupied then resource level is being decreased at a rate $\beta_{0}$.

Besides the change of resource level, the resource is being distributed in the field at a rate $\varepsilon$.

Finally, the resource field dynamics is defined by the equation

$$
u^{t+\tau}=u^{t}+\tau\left(\varepsilon \Delta u^{t}+\beta u^{t}-\gamma\left(u^{t}\right)^{2}+\zeta\right)
$$

where $u^{t}=u(x, y, t)$,

$$
\begin{gathered}
\Delta u^{t}=\frac{u_{x-1, y}^{t}+u_{x, y-1}^{t}-4 u_{x, y}^{t}+u_{x+1, y}^{t}+u_{x, y+1}^{t}}{h^{2}} \\
u(x, y, 0)=s_{m} p_{m}(x, y, 0) \\
\beta(x, y, t)=\beta_{m} p_{m}(x, y, t)-\beta_{f} p_{f}(x, y, t)-\beta_{0} \\
\gamma(x, y, t)=\gamma_{0}+\left(\gamma_{m}-\gamma_{0}\right) p_{m}(x, y, t) \\
\zeta(x, y, t)=\zeta_{m} p_{m}(x, y, t) .
\end{gathered}
$$

The constant $h$ is a size of lattice cell and $\tau$ is a time teak. 


\subsection{Computer simulation of interaction process based on the resource consumption}

We shall investigate a random distribution of $53 \mathrm{f}$-agents - the color is white and $47 \mathrm{~m}$-agents - the color is black, as shown in figure 3 , on the right.

Environment consists of 2500 locations arranged on a $50 \times 50$ lattice with the resource level at every site initially. The resource score is highest at the peaks in the field around the cell occupied by $m$-agent - where the color is most black - and falls off in a series of terraces.

Describe the simulation process in dynamics. The $f$-agent is moving on the field according to movement rule. Every time he consumes the resource in the cell occupied by himself. The $f$-agent go to the cell with maximum value of resource. The $m$-agent is accumulating the resource. It is being distributed on the resource field according to the rule of resource change on field. When the $f$-agent occupies the nearest cell around $m$-agent then the interaction process is realized.

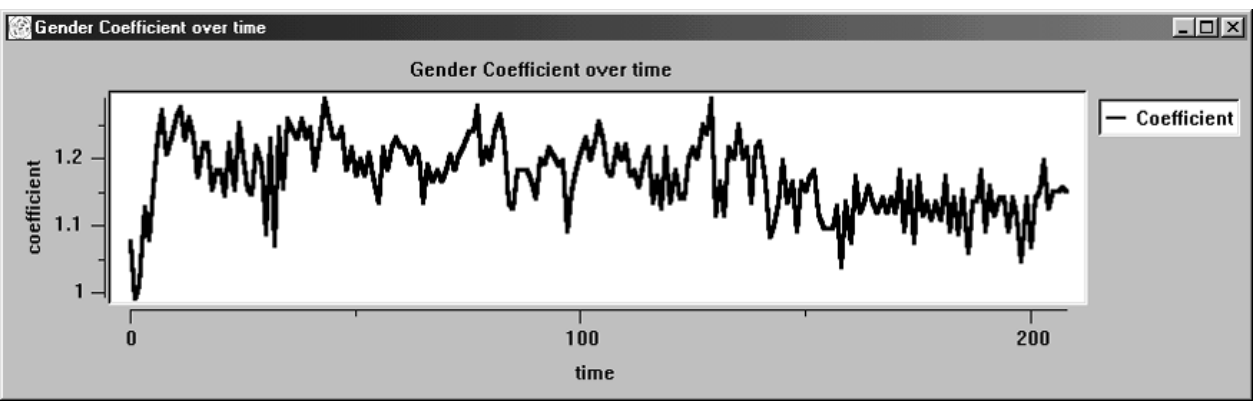

Fig. 5. Gender Interaction Coefficient value dynamics

On the figure 5 is presented the graph of Gender Interaction Coefficient value dynamics. $\mathcal{G}$ is fluctuating in interval $[1,1.3]$.

\section{Simulation results}

\subsection{The interaction types}

Under the computer simulation with various initial data, we reveal three types of agent interaction. These types is characterized by a subject-object interaction coefficient $\mathcal{K}$. There are three types of interaction:

- first type - stable. It is realized when rate of resource accumulation approximately equals the rate of resource consumption $(\mathcal{K} \approx 1)$;

- second type - unstable $(\mathcal{K} \ll 1)$;

- third type - multi-contact $(\mathcal{K} \gg 1)$. 
Under first interaction type, the contact process is a stable. In this case, the one subject is interacting with one object at long time. The unstable interaction is the contact process, realizing at short time. Under third interaction type, the one subject can interact with some objects at long time. In the first model the coefficient is defined by $\mathcal{K}=\beta / \alpha$, and in the second model $-\mathcal{K}=\beta_{m} / \beta_{f}$.

\subsection{The Gender Interaction Coefficient}

Although the agents are constantly moving and number of interaction is changed in artificial society, but the main characteristic of interaction process is staying on the fixed level. Such characteristic in our models is the Gender Interaction Coefficient. From the observe of coefficient value dynamics (see figures 4, 5) we have got the following results:

- in the first model, $\mathcal{G}$ is fluctuating about 0.75 . In this case the males have more activity than females for searching the interaction partner;

- in the second model, $\mathcal{G}$ is fluctuating about 1.15 . Here the females play the leader role on the amount of interaction.

\section{Conclusions}

Analyzing the results of investigation, we can make the following conclusions:

- the particularity of gender interaction modelling is the consideration of two agents' type with various sex. Therefore the gender relations differ from the interpersonal interactions by the competition of agents into group of its sex and the search of interaction partner, having opposite sex;

- defining the process of female's and male's interaction on base of subjectobject relations influence on the contact intensity. The agent is more active in the contact process when he is the interaction object;

- considering the gender as intrigue, we can say that the gender equality is a possible, if the female or male will acting not only as an object but also as a subject of interaction.

The research enables to define the influence of gender structure on society dynamics. It gives the possibility for studying the interconnection and the controlling factors of socium.

\section{References}

1. Epstein, J.M., Axtell. R.: Growing Artificial Societies: Social Science from the Bottom Up. Brookings Institution Press, Washington (1996)

2. Guts, A.K., Korobitsin, V.V., Laptev, A.A., Pautova, L.A., Frolova, J.V.: Mathematical Models of Social Systems. Omsk State University Press, Omsk (2000)

3. Haleeva, I.I.: Gender as Cognition Intrigue. Moscow State Linguistic University Press, Moscow (2000)

4. Parsons, T.: Social Structure and Personality. Free Press, New York (1964) 